\title{
Convolutional Neural Network Model in Machine Learning Methods and Computer Vision for Image Recognition: A Review
}

\author{
${ }^{1}$ R.M.Q.R.Jaapar, ${ }^{2}$ M.A.Mansor \\ ${ }^{1}$ Industrial Electronic, German Malaysian Institute, Kajang, Selangor Malaysia \\ ${ }^{2}$ Faculty of Engineering Technology, Universiti Malaysia Pahang, Malaysia
}

Correspondence Author: M.A.Mansor, Faculty of Engineering Technology, Universiti Malaysia Pahang, Malaysia

Received date: 23 October 2018, Accepted date: 15 November 2018, Online date: 25 December 2018

Copyright: (C) 2018 R.M.Q.R.Jaapar et al. This is an open-access article distributed under the terms of the Creative Commons Attribution License, which permits unrestricted use, distribution, and reproduction in any medium, provided the original author and source are credited.

\begin{abstract}
Recently, Convolutional Neural Networks (CNNs) are used in variety of areas including image and pattern recognition, speech recognition, biometric embedded vision, food recognition and video analysis for surveillance, industrial robots and autonomous cars. There are a number of reasons that convolutional neural networks (CNNs) are becoming important. Feature extractors are hand designed during traditional models for image recognition. In CNNs, the weights of the convolutional layer being used for feature extraction in addition to the fully connected layer are applied for classification that are determined during the training process. The objective of this paper is to review a few learning machine methods of convolutional neural network (CNNs) in image recognition. Furthermore, current approaches to image recognition make essential use of machine learning methods. Based on twenty five journal that have been review, this paper focusing on the development trend of convolution neural network (CNNs) model due to various learning method in image recognition since 2000s, which is mainly introduced from the aspects of capturing, verification and clustering. Consequently, deep convolutional neural network (DCNNs) have shown much successful in various machine learning and computer vision problem because it significant quality gain at a modest increase of computational requirement. This training method also allows models that are composed of multiple processing layers to learn representation of data with multiple levels of abstraction.
\end{abstract}

Key words: Neural Network, Image Recognition, Learning Machine, Computer Vision

\section{INTRODUCTION}

Nowadays, Convolutional Neural Networks (CNNs) have shown that much successful in various machine learning and computer vision problems [1], [2]. Moreover, Convolutional Neural Networks (CNNs) are used in variety of areas in recognition and robotic field. There are a number of reasons that convolutional neural networks (CNNs) are becoming important. In the meantime convolutional neural networks (CNNs) have been applied with great success to the image recognition of objects in the air from the past few years [3]. From the sides of validity it is more naturally and simply in term of capturing an image.

CNNs assumes the nature of the image, such as static images and where pixel dependencies are. Thus, compared to standard feed-forward neural networks with similarly-sized layers, CNNs have much fewer connections and parameters and that will make they are easier to train, while by theoretically the best achievement and may be just a little worse [4]. In addition, the capacity of CNNs can be controlled by varying their depth and breadth due to their architecture performance.

Consequently, the typical architecture of CNNs is a multilayer stack of simple modules such as convolutional layer, pooling layer and fully-connected layer. Starting with the raw input, each module transforms the representation at one level into a higher and more abstract level [5]. Meanwhile, for recognition tasks, higher ranking of dataset representations an increase aspects of the input which are important for discrimination and limit unrelated of variations. From that, the latest technique of image recognition is introduce.

Only a year later, the deep learning technique based on convolutional neural network has achieved great performance improvement in large-scale image classification tasks and set off the upsurge of deep learning besides it is a new hotspot in the domain of pattern recognition [6]. It allows a model that consists of multiple processing layers to study data representation with various levels of abstraction. Furthermore, in deep learning techniques, besides data formation, transfer learning is useful when someone wants to train on their own dataset for various reasons, for example, the dataset may not be enough to train the full neural net and cause problems in transfer learning [7].

Specifically, transfer learning may be used to take a pre-trained Deep Neural Networks, replacing the fully-connected layers (and potentially the last convolutional layer) and training those layers on the related dataset. Nevertheless, it has been observed that deep neural networks (DNNs) easily suffer from over fitting with small samples. In this review, the technique of machine learning is important to ensure the quality and efficiency of image in terms of capturing, verification and clustering that want to train more effectiveness especially in image recognition by using the accurate learning machines method

The objective of this paper is to study the various machine learning required to apply in the CNNs mode depending on the data and occasionally in the state they want to use. Literature records show that there have been a number of valuable reviews on Machine Learning techniques. However, review on differences of learning machine to identify images and systems that train data is scarce. Therefore, based on twenty five journals that have been reviewed, this paper focuses on the image recognition in terms of capturing, verification and clustering. 


\section{LITERATURE SURVEY}

This part will describe and expose better solution or technique for image recognition after some related journals, conferences, book chapters and websites reviewed. Several solutions of image recognition acclaimed by other authors though have certain limitations of their proposed methods. All overview of image recognition in Convolution Neural Networks was compiled and discussed.

The first approach for the technique to train a dataset is Deep Learning Neural Networks. That will train by two systems which are Deep IDentificationverification features (DeepID2) and Image-Net.

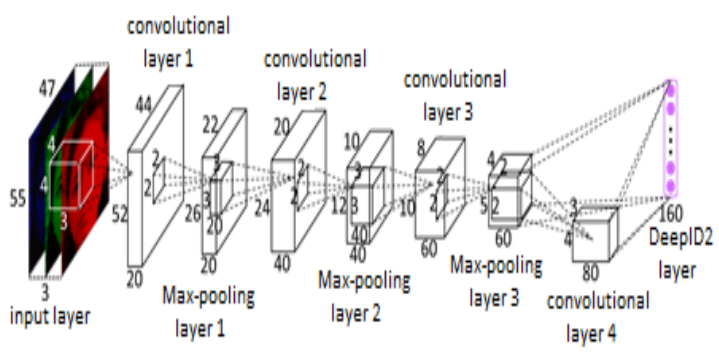

Fig. 1: The ConvNet structure for DeepID2 extraction [8].

For DeepID2 that are learned with carefully designed, the image identification task increases the inter-personal variations by drawing DeepID2 extracted from different identities apart, while the image verification task reduces the intra-personal variations by pulling DeepID2 extracted from the same identity together where both of which are needed to image recognition.

It has shown that the effect of the image identification and verification supervisory signals on deep feature representation coincide with the two aspects of constructing ideal features are: 1) increasing inter-personal variations, 2) reducing intra-personal variations and the combination of the two supervisory signals lead to significantly better features than either one of them.

While, the other system is Image-Net also applied to deep learning technique that will trained to directly optimize the embedding itself, rather than an intermediate bottleneck layer. In this system directly learns a mapping from face images to a compact Euclidean space where distances directly correspond to a measure of face similarity. Once this space has been produced, tasks such as image recognition, verification and clustering can be easily implemented using standard techniques with Face-Net embedding as feature vectors [9]. The main features of this architecture is the improved utilization of the computing resources inside the network. It was increased the depth and width of the network while keeping the computational budget constant [10]. Image-Net provides such a large image of dataset, which has over 15 million labeled high resolution images belonging to roughly 22,000 categories [11]. The images of each concept are qualitycontrolled and human-annotated.

The main advantage of this method is a significant quality gain at a modest increase of computational requirements compared to shallower and narrower architectures [12]. As define and detail the space of fully convolutional networks, illuminate the application for a solid prediction tasks and connecting with previous models that adjust the contemporary classification networks into deep convolutional networks and move the learned representation with adjustment to the segmentation task. Then define a skip architecture that combines semantic information from a deep and coarse layer with appearance information of superficial, shallow layers to produce accurate and detailed segmentations.

For the second technique of image recognition is Double Local Binary Pattern (DLBP) that introduced due to an integrated automatic system that often involves in two components: 1) peak expression frame detection and 2) expression feature extraction [13]. In comparison with the image-based expression recognition system, the video-based recognition system often performs online detection which prefers low-dimensional feature representation for costeffectiveness. In fact, the double local binary pattern (DLBP) is to detect the peak expression frame from the video due to DLBP technique has a much lowerdimensional size and can successfully reduce detection time.

This technique involved to handle the illumination variations in DLBP and Logarithm-Laplace (LL) domain is further proposed to get a more robust facial feature for detection. The Taylor expansion theorem is employed in the system for the first time to extract facial expression feature and to obtain an effective facial feature from the Taylor Feature Map. DLBP [14] is a representative feature extraction technique which has been widely used in face recognition [15], facial analysis [16], and texture classification [17], and many other tasks [18].

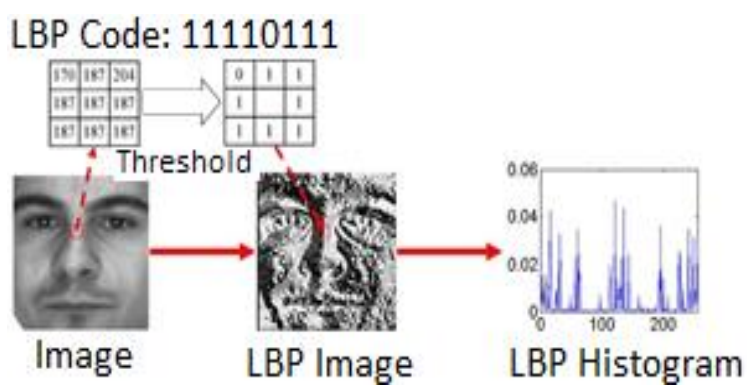

Fig. 2: Illustration of the feature extraction process for LBP [19].

However, the recognition rate of the DLBP based on feature extraction method drops sharply when the set coming from different datasets with the training data showing that deep learning model is easily over-fitted on the training data. On the contrary, the DLBP technique still has an acceptable recognition performance in image even when the gallery set and the probe set coming from different datasets [20].

The other technique in this paper is the Bilinear Convolutional Neural Networks (BCNNs) that is used by Tanenbaum and Freeman to model two factor variations such as "style" and "content" for images [21]. This technique is the state-of-the-art in fine-grained image recognition and fails in individual the categories with indirect visual differences [22]. These networks represent the image as an outside product that is collected from the features obtained from two CNNs and capture local feature interactions in a transnationally invariant way. 


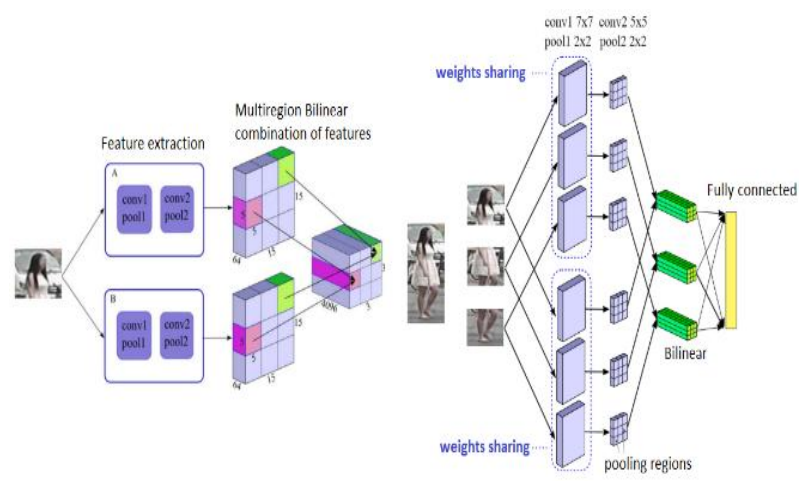

Fig. 3: The proposed architecture for person re-identification [23].

A systematic analysis of these networks show that the bilinear features are highly redundant and can be reduced by an order of magnitude in size without significant loss in accuracy. Second are also effective for other image classification tasks such as texture and scene recognition and third it can be trained from scratch on the Image-Net dataset offering consistent improvements over the baseline architecture.

Hence, BCNNs compare constructively to traditional CNNs architectures in terms of speed since the replacement of several fully connected layers with a bilinear pooling layer and a linear layer. Moreover, BCNNs are related to order less texture representations built on deep features but can be trained in an end-toend manner on most accurate model shows that obtains $84.1 \%, 79.4 \%, 84.5 \%$ and $91.3 \%$ per image accuracy for the Caltech-UCSD birds [24], NABirds [25], FGVC aircraft [26], and Stanford cars [27] that were dataset respectively runs at 30 frames-per-second on a NVIDIA Titan X GPU.

The main advantage of BCNNs is that they require image labels only and can be easily applied to different fine-grained datasets. This technique is prefer to detect an image in deep of water. In particular, BCNN allows to retain some spatial information and to extract more complex features, while increase the number of parameters over the baseline CNNs without over-fitting [28]. That have been demonstrated notable gap between the performance of the BCNNs and the performance of the standard CNNs [29].

Another comprehensive technique on Multiple attributes (MA) has been conducted by Liu, Xu and Chiu [30]. The common adopted methods from gender recognition system that only focus on the face image to make gender identification was include using LBP and PCA for feature extraction and dimension reduction, Euclidean and Manhattan classifiers are used [31], and using Gabor Filters to extract features, Support Vector Machines, k-NN classifiers were tested [32], and using Discrete Cosine Transform (DCT) to extract features, K-nearest neighbor classifier (KNN) classifiers are used [33].

To gain a better understanding on the attributes such as long hair, short hair, with or without tie, skirt and so on which are located on face, upper-body and lowerbody, three feature blocks helps distinguish between men and women as in Table 1 shown [30].

Table 1: The accuracy of each pair of attributes.

\begin{tabular}{|c|c|c|c|}
\hline Parts & Attributes Classification & Weighting & Accuracy \\
\hline \multirow{6}{*}{ Face } & female face & 0.3 & \multirow{2}{*}{$90 \%$} \\
\hline & male face & 0.3 & \\
\hline & long hairs & 0.2 & \multirow{2}{*}{$92.5 \%$} \\
\hline & short hair & 0.2 & \\
\hline & no bread & 0 & \multirow{2}{*}{$87.5 \%$} \\
\hline & bread & 0.15 & \\
\hline \multirow{4}{*}{ Upper body } & womens clothing & 0.1 & \multirow{2}{*}{$89.04 \%$} \\
\hline & no womens clothing & 0.05 & \\
\hline & no tie & 0 & \multirow{4}{*}{$85.71 \%$} \\
\hline & tie & 0.2 & \\
\hline \multirow{4}{*}{ Lower body } & skirt & 0.2 & \\
\hline & no skirt & 0.05 & \\
\hline & hot pants & 0.2 & \multirow{2}{*}{$80 \%$} \\
\hline & no hot pants & 0.05 & \\
\hline
\end{tabular}

This Finding is consistent with the earlier study by Liu, Xu and Chiu. Another innovative research was conducted by them regarding the gender recognition enhancement by assign weights to each of the different attributes wi , and calculate the male scores and the female scores. Finally, the result of gender identification is obtained by comparing the score. In an innovative work by Liu and his co-researcher, by the distribution of attribute weights to obtain gender scores, the accuracy of gender recognition will achieve a more significant effect.

\section{COMPARISON OF IMAGE RECOGNITION TECHNIQUES}

The aforementioned papers discussed several techniques that was applied in Convolutional Neural Network to train the learning for image recognition. Then, in this section, gives a brief about the comparison on that learning machines due to the conventional models for image recognition use carefully hand crafted low level features. The features do achieve good performance for some specific data and tasks but effective features require specialized knowledge and most of them are limited in generalization capability during the training process [34].

From the results and achievement data, shows that the CNNs not only give the best performance compared to other detection algorithms, even outperform humans in cases such as classifying objects into fine-grained categories same like breed of dog or species of bird [35]. In addition, the best possible correct detection rates (CDRs) have been achieved using CNNs. For example, CNNs have achieved a CDR of $99.77 \%$ using the MNIST database of handwritten digits [36], a CDR of $97.47 \%$ with the NORB dataset of 3D objects [37], and a CDR of 97.6\% on 5600 images of more than 10 objects [38]. Approved that CNNs is more commercial using in machine learning methods that applied in computer vision.

Because of that, various system in machine learning technique existed to train the deep learning especially in image recognition due to require careful understanding of the image such as: Alex-Net [39], the VGG net [40], and GoogLe-Net [41] compared with the two technique that have mentioned before. 
Citation: R.M.Q.R.Jaapar and M.A.Mansor, 2018. Convolutional Neural Network Model in Machine Learning Methods and Computer Vision for Image Recognition: A Review. Journal of Applied Sciences Research., 14(6): 23-27. DOI: 10.22587/jasr.2018.14.6.5

Table 2: Comparison four types of machine learning technique

\begin{tabular}{|c|c|c|c|}
\hline Machine Learning Technique & Advantage & Focused & System \\
\hline Deep Learning & $\begin{array}{c}\text { significant quality gain at a modest } \\
\text { increase of computational } \\
\text { requirements compared to shallower } \\
\text { and narrower architectures }\end{array}$ & $\begin{array}{c}\text { recognition, verification and } \\
\text { clustering in image } \\
\text { implemented }\end{array}$ & $\begin{array}{c}\text { 1)DeepID2 } \\
\text { 2)Image-Net }\end{array}$ \\
\hline DLBP & $\begin{array}{c}\text { Detect the peak expression frame } \\
\text { from the video due to DLBP } \\
\text { technique has a much lower- } \\
\text { dimensional size and can successfully } \\
\text { reduce detection time }\end{array}$ & $\begin{array}{c}\text { Tideo-based recognition } \\
\text { system }\end{array}$ & $\begin{array}{c}\text { 1)Tanenbaum } \\
\text { 2) Freeman }\end{array}$ \\
\hline BCNN & $\begin{array}{c}\text { Require image labels only and can be } \\
\text { easily applied to different fine- } \\
\text { grained datasets }\end{array}$ & fine-grained image recognition & 1) HOG \\
\hline Multipleattributes (MA) & $\begin{array}{c}\text { Proprietary method of whole-body } \\
\text { identification to make the best of } \\
\text { multi-attribute (MA) recognition. } \\
\text { Besides facial information, it also } \\
\text { knows the type of clothing used }\end{array}$ & $\begin{array}{c}\text { face image to make gender } \\
\text { identification }\end{array}$ & 2) SVM \\
\hline
\end{tabular}

Table 2 shows a comparison between three training machine learning that are used to train the data in image recognition for Convolutional Neural Networks. Three aspects where advantage, focused and system that have been used in training session. Additionally, by deep research of this techniques will able to learn an effective feature of variety computer vision tasks. In fact, appreciations to its deep architecture and large learning capacity due to some deep neural networks even get close to human performance on recognition tasks. It helps to reduce some issues when its use computer vision due to understanding the concept of train the data.

Moreover, a number of well-established problems in computer vision have recently benefited from the rise in deep learning as feature representations or classifiers. For instance, DeepID2 [42] and DeepID2+ [43] utilize the idea of joint face identification-verification to reduce intra-personal variations which leads to a significant improvement on face recognition accuracy.
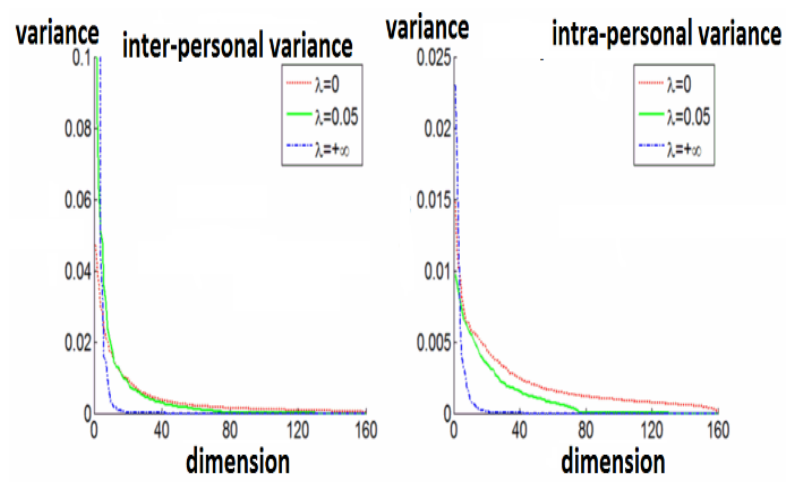

Fig. 4: Spectrum of eigenvalues of the inter-personal and intra-personal scatter matrices [42].

The corresponding eigenvectors represent different patterns of variation. Both the magnitude and the diversity of variations are important in recognition. If all the variations of the feature focus on a small number of eigenvectors, it indicates that the variety of intra or inter-personal variants is low. The characteristics studied with $\lambda=0,0.05$, and $\infty$, respectively. The characteristic variation of each $\lambda$ is given normalized by the corresponding variance of the mean feature.

\section{CONCLUSIONS}

This paper reviews several methods in terms of recognition, verification and clustering. The results, shows that the deep learning technique is incredibly effective in extensive dataset. Deep learning has successful in the system of Convolutional Neural Networks based on image implemented due to more than twenty journals that have been reviewed, deep learning give the best performance in image recognition problems and even outperform humans in certain cases. Further, the deep learning able to train the models directly on the full sized of images and even though sometimes the data use is large amount of dataset [44].

Besides, the technique of deep learning also recommended for apply at any place including deep surface for example to underwater image, winding space or else. In addition, deep learning contribute positive impact in image processing and computer vision as well as the impact is unquestionable. New application of deep learning can be develop by conducting the research and understand its limitation in order to produce the best outcome.

In conclusion, based on related aspect that have been reviewed, deep learning is the most effective technique ever in data training due to easy to handle and close to human way when said about image recognition. As a result of the reading, deep learning is widely and mostly used for an image in terms of recognition, identification, verification and classification. Thus, this technique is more convenience in term of understanding or to train the data in image recognition of computer vision.

\section{REFERENCES}

[1] D. Mishkin, N. Sergievskiy, and J. Matas, 2016. "Systematic evaluation of CNN advances on the ImageNet," ArXiv e-prints.

[2] J. Tang, D. Wang, Z. Zhang, L. He, J. Xin, and Y. Xu, 2017. "Weed identification based on k-means feature learning combined with convolutional neural network," Computers and Electronics in Agriculture, 135:63-70,

[3] Lecun Y, Bengio Y and Hinton G, 2015. Deep learning, Nature, 521(7553):436-444

[4] Krizhevsky A, Sutskever I and Hinton G E, 2012. ImageNet classification with deep convolutional neural networks, International Conference on Neural Information Processing Systems, Curran Associates Inc, 10971105

[5] Moacir A. Ponti, Leonardo S. F. Ribeiro, Tiago S. Nazare Tu Bui, John Collomosse, "Everything you wanted to know about Deep Learning for Computer Vision but were afraid to ask,", Patterns and Images Tutorials, 2474-0705/17

[6] Leilei Jin, Hong Liang. Deep Learning for Underwater Image Recognition in Small Sample Size Situations"IEEE 978-1-5090-5278-3/17/2017.

[7] A.Ahmed, T.Hai, M. Ahmed, "Review of Deep Convolution Neural Network in Image Classification", 2017 International Conference on Radar, Antenna, Microwave, Electronics, and Telecommunications, IEEE 978-1-5386-3849-1/17-2017

[8] Yi Sun, Xiaogang Wang, Xiaoou Tang, 2014. Deep Learning Face Representation by Joint Identification-Verification. 1406.47731 
[9] Florian Schroff, Dmitry Kalenichenko, James Philbin, 2015. "FaceNet: A Unified Embedding for Face Recognition and Clustering". In IEEE Conf. on CVPR

[10] Christian Szegedy, Wei Liu, Yangqing Jia, Pierre Sermanet. "Going Deeper with Convolutions". In IEEE Conf. on CVPR2015.

[11] J. Deng, W. Dong, R. Socher, L. J. Li, K. Li and L. Fei-Fei, Imagenet : a large-scale hierarchical image database, in: 2009 IEEE Conference on Computer Vision and Pattern Recognition, CVPR2009, IEEE, Miami, FL, USA, 2009, pp.248-255.

[12] Florian Schroff, Dmitry Kalenichenko, James Philbin. "FaceNet: A Unified Embedding for Face Recognition and Clustering”. In IEEE Conf. on CVPR2015.

[13] B. Hatipoglu and C. Köse, "Gender Recognition from Face Images Using PCA and LBP, "International Conference on Electrical and Electronics Engineering, pp. 1258-1262, 26-28. B. Yang and S. Chen, "A comparative study on local binary pattern (LBP) based face recognition: LBP histogram versus LBP image," Neurocomputing, vol. 120, no. 10, pp. 365-379, 2013.

[14] J. Ren, X. Jiang and J. Yuan, "LBP Encoding Schemes Jointly Utilizing the Information of Current Bit and Other LBP Bits," IEEE Signal. Process. Lett., vol. 22, no. 12, pp. 2373-2377, Dec. 2015.

[15] Y. Tong, R. Chen, Y. Cheng, "Facial expression recognition algorithm using LGC based on horizontal and diagonal prior principle," Optik - International Journal for Light and Electron Optics, vol. 125, no. 16, pp. 4186- 4189, 2014.

[16] Z. Guo, L. Zhang, and D. Zhang, "Rotation invariant texture classification using LBP variance (LBPV) with global matching," Pattern Recogn., vol. 43, no. 3, pp. 706-719, 2010.

[17] X. Wang, T.X. Han, S. Yan, “An HOG-LBP human detector with partial occlusion handling,” in Proc. ICCV, Kyoto, Japan, 2009, pp.32-39.

[18] J. Zhang, et. al, "Boosted local structured HOG-LBP for object localization," in Proc. CVPR, Colorado Springs, CO, USA, 2011, pp.1393-1400.

[19] Yuanyuan Ding, Qin Zhao, Baoqing Li, Xiaobing Yuan." Facial Expression Recognition from Image Sequence based on LBP and Taylor Expansion". DOI 10.1109/ACCESS.2017.2737821, IEEE Access.

[20] J. B. Tenenbaum and W. T. Freeman. Separating style and content with bilinear models. Neural computation, 12(6):1247-1283, 2000. 3

[21] Guangjian Zheng, Min Tan, Jun Yu, Qing Wu, Jianping Fan' "fine-grained image recognition via weakly supervised click data guided bilinear cnn model". IEEE international conference on multimedia and expo (icme) 2017 july 2017.

[22] U.Evgeniya, G.Yaroslav, L.Victor. "Multi-region Bilinear Convolutional Neural Networks for Person Re-Identification", IEEE 978-1-5386-2939-0/17-2017.

[23] C. Wah, S. Branson, P. Welinder, P. Perona, and S. Belongie. The Caltech-UCSD Birds-200-2011 Dataset. Technical Report CNS-TR2011-001, CalTech, 2011. 1, 2, 6, 8, 12

[24] G. Van Horn, S. Branson, R. Farrell, S. Haber, J. Barry, P. Ipeirotis, P. Perona, and S. Belongie. Building a bird recognition app and large scale dataset with citizen scientists: The fine print in finegrained dataset collection. In The IEEE Conference on Computer Vision and Pattern Recognition (CVPR), 2015. 1, 6, 7

[25] S. Maji, E. Rahtu, J. Kannala, M. Blaschko, and A. Vedaldi. Fine-grained visual classification of aircraft. arXiv preprint arXiv:1306.5151, 2013. 1, 6, 8

[26] J. Krause, M. Stark, J. Deng, and L. Fei-Fei. 3d object representations for fine-grained categorization. In 3D Representation and Recognition Workshop, at ICCV, 2013. 1, 6, 8

[27] U.Evgeniya, G.Yaroslav, L.Victor. "Multi-region Bilinear Convolutional Neural Networks for Person Re-Identification”, IEEE 978-1-5386-2939-0/17-2017.

[28] D. Yi, Z. Lei, and S. Z. Li. Deep metric learning for practical person re-identification. arXiv prepzrint arXiv:1407.4979, 2014.

[29] Hsuan-Hung Liu, Sendren Sheng-Dong Xu, Chung-Cheng Chiu and Sheng-Yi Chiu, "Gender Recognition Technology of Whole Body Image", IEEE International Conference on Consumer Electronics - Taiwan (ICCE-TW), 2017.

[30] B. Hatipoglu and C. Köse, "Gender Recognition from Face Images Using PCA and LBP," International Conference on Electrical and Electronics Engineering, pp. 1258-1262, 26-28 Nov. 2015

[31] Ignat and M. Coman, "Gender Recognition with Gabor Filters," EHealth and Bioengineering Conference (EHB), pp. 1-4, 19-21 Nov. 2015.

[32] M. Nazir, M. Ishtiaq, A.Batool, M. A. Jaffar and A. M. Mirza, "Feature Selection for Efficient Gender Classification," World Scientific and Engineering Academy and Society (WSEAS), pp. 70-75, 13-15 June. 2010.

[33] Qin, H., Li, X., Liang, J., Peng, Y., and Zhang, C, DeepFish: Accurate Information Processing Systems, Curran Associates Inc, 2012 , pp. 10971105.

[34] Russakovsky, O. et al. 2014. "ImageNet Large Scale Visual Recognition Challenge.” International Journal of Computer Vision..1007/s11263-015-0816-y

[35] Ciresan, Dan, Ueli Meier, and Jürgen Schmidhuber. 2012. "Multi-column deep neural networks for image classification." IEEE Conference on Computer Vision and Pattern Recognition, 2012.

[36] Ciresan, Dan, Ueli Meier, Jonathan Masci, Luca M. Gambardella, and Jurgen Schmidhuber. 2011. "Flexible High Performance Convolutional Neural Networks for Image Classification.” Proceedings of the Twenty-Second International Joint Conference on Artificial Intelligence-Volume Two: 1237-1242. Retrieved 17 November 2013.

[37] Lawrence, Steve, C. Lee Giles, Ah Chung Tsoi, and Andrew D. Back. 1997. "Face Recognition: A Convolutional Neural Network Approach." IEEE Transactions on Neural Networks, Volume 8; Issue 1

[38] Krizhevsky, I. Sutskever, and G. E. Hinton. Imagenet classification with deep convolutional neural networks. In NIPS, 2012. 1, 2, 3, 5

[39] K. Simonyan and A. Zisserman. Very deep convolutional networks for large-scale image recognition. CoRR, abs/1409.1556, 2014. 1, 2, 3, 5

[40] C. Szegedy, W. Liu, Y. Jia, P. Sermanet,S. Reed, D. Anguelov, D. Erhan, V. Vanhoucke, and A. Rabinovich. Going deeper with convolutions. CoRR, abs/1409.4842, 2014. 1, 2, 3, 5

[41] Y. Sun, X. Wang, and X. Tang, "Deep learning face representation by joint identification-verification, ” in Proc. NIPS, Montréal, Quebec, Canada, 2014, pp.1988-1996.

[42] Y. Sun, X. Wang and X. Tang, "Deeply learned face representations are sparse, selective, and robust," in Proc. CVPR, Boston, MA, 2015, pp. 2892-2900.

[43] Y. Sun, X. Wang and X. Tang, "Hybrid deep Learning for Face Verification", IEEE transactions on pattern analysis and machine intelligence, vol. 38; Issue $10,2016$. 